\title{
Special Section Guest Editorial: Perceptually Optimized Imaging
}

\author{
Shuhang Gu, ${ }^{a}$ Kede Ma, ${ }^{b}$ and Radu Timofte \\ ${ }^{a}$ The University of Sydney, Sydney, Australia \\ ${ }^{\mathrm{b}}$ The City University of Hong Kong, Hong Kong, China \\ ${ }^{\mathrm{c}}$ ETH Zurich, Zurich, Switzerland
}

The Special Section on Perceptually Optimized Imaging focuses on the latest research work in the field of perceptually oriented image restoration and enhancement. The four accepted papers cover four different topics of perceptually optimized imaging, including video stabilization, single image super-resolution, single-pixel imaging, and image dehazing.

In the field of video stabilization, Ren et al. proposed a virtual sphere model to stabilize video frames by projecting each frame onto the sphere and performing corrective rotations.

Chudasama et al. proposed a computationally efficient approach for the single image superresolution task. The proposed method introduces a novel design of residual block and utilizes the generative adversarial network loss to generate visually plausible solutions.

On single pixel imaging, Shin et al. presented a design of sensing patterns to obtain image information by the spatially variant resolution technique. The resulting method reduces the measurements by prioritizing the resolution in the region of interest and successfully achieves the programmable imaging while adaptively trading off image quality and the number of measurements.

In the field of image dehazing, Sun et al. proposed a real-time image dehazing algorithm based on dark channel prior and fast weighted guided filtering. The resulting method can effectively remove the halo artifacts, correct the color deviation, and retain more details in the images.

All these papers show that perceptually optimized imaging is a rich research area. While deep neural networks have achieved tremendous success in the last several years, conventional priors still play important roles in many tasks. 